\title{
Das Bundesstatistikgesetz 2000 aus der Sicht des universitären Statistikers
}

\author{
Peter Bauer ${ }^{1}$ und Peter Hackl ${ }^{2}$ \\ ${ }^{1}$ Institut für Medizinische Statistik, Universität Wien \\ ${ }^{2}$ Institut für Statistik, Wirtschaftsuniversität Wien
}

\begin{abstract}
Zusammenfassung: Der universitäre Statistiker hat naturgemäß besonderes Interesse an methodischen Fragen, die allerdings für die Sicherung der Qualität der Ergebnisse von statistischen Analysen ganz allgemein von großer Relevanz sind. In diesem Sinn wurde bereits im Herbst 1997 aus Anlaß einer Podiumsdiskussion argumentiert und entsprechende Wünsche an ein künftiges Bundesstatistikgesetz geäußert (siehe den Beitrag eines der Autoren in „Wünsche an ein Österreichisches Bundesstatistik-Gesetz“, Österreichische Zeitschrift für Statistik, 26 (1997), 71-84). Ein zweites Thema, das für die Arbeit universitärer Statistiker von Wichtigkeit ist, betrifft die Möglichkeit des Zugriffs auf Daten und statistische Ergebnisse aus dem Bereich der Amtlichen Statistik. Im vorliegenden Beitrag soll dargelegt werden, inwieweit das Bundesstatistikgesetz 2000 (im Folgenden mit BSG 2000 abgekürzt) den Vorstellungen des universitären Statistikers entspricht und welche Wünsche es offen läßt.
\end{abstract}

\section{Statistische Methodik und Amtliche Statistik}

Welche negativen Folgen qualitativ mangelhafte Statistiken verursachen, braucht für den Leser einer statistischen Zeitschrift nicht ausgeführt werden. Geht es um Ergebnisse der Amtlichen Statistik, so muß vermutet werden, daß mangelhafte Statistiken zur Folge haben, daß wichtige Entscheidungen in Politik, Wirtschaft und öffentlicher Verwaltung auf einer ungeeigneten Grundlage gefällt werden. Diese Entscheidungen haben aber, wie man weiß, in der Regel für alle Bürger weitreichende wirtschaftliche und soziale Konsequenzen. Die Qualität von statistischen Ergebnissen wird wesentlich von der verwendeten statistischen Methodik bestimmt. Wichtige Determinanten sind das Design der Datenerhebung, darunter die Responserate, und die Verfahren der statistischen Analyse. Die passende Wahl der anzuwendenden statistischen Methodik ist von nicht zu unterschätzender Bedeutung.

Die Auswahl der statistischen Methodik ist eine Tätigkeit, die auf Grund ihrer Vielfalt und Komplexität wissenschaftlichen Charakter hat. In diesem Sinn verlangt auch die Verordnung (EC) Nr. 322/97 des Rates vom 17. Februar 1997 über Gemeinschaftsstatistiken „die Heranziehung wissenschaftlicher Kriterien bei der Wahl der Quellen, Methoden und Verfahren" und die Offenlegung von Erhebungsumfang, Methodik, Verfahren und Quellen, um die Zuverlässigkeit der Daten zu verbessern. Ein zentraler Wunsch der Teilnehmer der Podiumsdiskussion im Herbst 1997 war es demnach, daß das künftige Bundesstatistikgesetz „den wissenschaftliche Charakter statistischer Methoden anerkennt“ und die Verpflichtung vorsieht, ,dem jeweiligen Stand der Wissenschaften gemäße Verfahren anzuwenden“. Diesen Wunsch erfüllt das BSG 2000 in hohem Maße. In Par. 22 
des BSG 2000 wird als Aufgabe der künftigen Bundesanstalt „Statistik Österreich“ das Erbringen ,,von Dienstleistungen wissenschaftlichen Charakters im öffentlichen Interesse“ definiert. In Par. 24 wird der Bundesanstalt „Statistik Österreich“ vorgeschrieben, „statistische Methoden und Verfahren nach international anerkannten wissenschaftlichen Grundsätzen und Standards“ anzuwenden. In den Erläuterungen des BSG 2000 wird in diesem Zusammenhang explizit darauf hingewiesen, daß mit diesem Grundsatz ,hochqualitative statistische Produkte“" angestrebt werden.

Darüber hinaus wurden weitere Maßnahmen vorgesehen, die geeignet sind, die Qualität der Ergebnisse der Amtlichen Statistik zu sichern:

- So wird in Par. 24 des BSG 2000 der Grundsatz postuliert, daß die Statistiken laufend auf (mögliche) Qualitätsverbesserungen überprüft werden.

- Der Par. 14 des BSG 2000 verlangt, daß die „Grundsätze der Objektivität und der Zuverlässigkeit“, aber auch ,,der Erheblichkeit, der Kostenwirksamkeit und der Transparenz“ zu befolgen seien und „eine möglichst hohe Kohärenz aller Statistiken“ anzustreben sei. Der Grundsatz der Objektivität wird gemeinsam mit dem der Unparteilichkeit bei der Erstellung der Statistiken auch in Par. 24 angesprochen.

- Im Par. 24 wird auch das Sicherstellen einer möglichst hohen Aktualität der Statistiken auferlegt, ein Qualitätsaspekt, der neben den inhaltlichen Qualitätsdimensionen nicht übersehen werden sollte.

Das Postulieren aller dieser Grundsätze, die wohl auch den Vergleich mit guten internationalen Regelungen standhalten, ist natürlich zu begrüßen. Das Postulieren von Grundsätzen stellt allerdings nicht sicher, daß diese auch zur Richtschnur für das Handeln der Akteure werden. Besondere Bedeutung für die Umsetzung dieser Grundsätze kommt daher folgenden Regelungen zu:

- Das BSG 2000 anerkennt im Par. 38 die Weisungsfreiheit der Amtlichen Statistik bei der Wahl der anzuwendenden statistischen Methoden und überträgt die Verantwortung dem fachlichen Leiter: „In allen wissenschaftlich methodischen Fragen ist er bei der Erfüllung der ihm obliegenden Aufgaben weisungsfrei.“

- Eine Maßnahme der Qualitätssicherung stellt die Einrichtung des Statistikrates dar, ein 15-köpfiges Gremium von Experten, die vorwiegend von Ministerien, der Österreichischen Nationalbank und Interessensvertretung entsandt werden, den aber auch ein im Fach Statistik habilitierter Wissenschafter angehören soll. Der Statistikrat soll in jährlichen Berichten beurteilen, inwieweit die oben skizzierten Grundsätze eingehalten werden, aber auch Empfehlungen und Stellungnahmen zu Arbeitsprogrammen und bestimmten technischen Fragen abgeben.

- Der Par. 19 des BSG 2000 sieht vor, daß zu statistischen Ergebnissen auch ,deren Konzepte, Definitionen und Erläuterungen“ veröffentlicht werden. Geht man davon aus, daß damit eine Darstellung der statistischen Methodik einschließlich Informationen zum Design der Erhebung und Analyse der Daten gemeint ist, so bedeutet 
das, daß dem Konsumenten wichtige Indikatoren der Qualität der statistischen Ergebnisse in die Hand gegeben werden. Dieses Offenlegen der wesentlichen Charakteristika des statistischen Produktes und die damit vorgeschriebene Transparenz der verwendeten Daten und Methoden ist ebenfalls ein als wirksames Instrument der Qualitätssicherung zu sehen.

Schließlich soll noch ein weiterer Aspekt angesprochen werden, der für die Qualität von statistischen Analysen von Relevanz ist. Für das Beschaffen von Daten eröffnet das BSG 2000 den Zugriff auf Daten der öffentlichen Verwaltung; Primärerhebungen sind nur dann zulässig, wenn Daten der öffentlichen Verwaltung nicht verfügbar sind. Durch diese für Österreich radikale Neuerung wird die Bundesanstalt „Statistik Österreich“ mit einer weiteren Aufgabe im Umgang mit Daten konfrontiert. Die Bundesanstalt wird eine Evidenthaltung von in der öffentlichen Verwaltung verfügbaren Daten und deren Qualitätscharakteristika aufzubauen und vermehrte Kompetenz für Stichprobenverfahren und für Methoden zum Verarbeiten von Daten aus unterschiedlichen Quellen zu entwickeln haben.

Alle diese Gegebenheiten des BSG 2000 haben auch eine inhaltliche Dimension. Das Erfüllen der Anforderungen des neuen Gesetzes wird wesentlich mehr auf statistische Methoden und die modernen Informationstechnologien spezialisierte Mitarbeiter erfordern, als bisher der Amtlichen Statistik zur Verfügung standen. Weiterbildung in statistischen Methoden sollte allen Mitarbeitern angeboten werden. Es sollte auch Platz für Mitarbeiter geben, die sich vorwiegend mit methodischen Fragen befassen und die auch Zeit für Erfahrungsaustausch mit Experten in- und ausländischer Ämter und der Universitäten haben.

\section{Zugang der Wissenschaft zu Daten der Amtlichen Sta- tistik}

Dem Zugang der Wissenschaft zu statistischen Daten ist ein eigener Paragraph des BSG 2000 gewidmet. Im Par. 31 wird allerdings eine Regelung getroffen, die diesen Zugang so sehr einschränkt, daß er vermutlich keine praktische Relevanz haben wird. Die Bundesanstalt ,Statistik Österreich“ ist verpflichtet, die Hauptergebnisse per Internet und unentgeltlich, Detailergebnisse in einer elektronischen Datenbank gegen Entgelt zugänglich zu machen (Par. 30). Die Ergebnisse sind so zu veröffentlichen, daß Rückschlüsse auf Angaben von Betroffenen ausgeschlossen werden können (Par. 19). Für wissenschaftliche Zwecke kann nun die Bundesanstalt „Statistik Österreich“ Zugang zu weiteren Daten einräumen. Allerdings müssen zwei Bedingungen zu erfüllen sein:

- Diese Daten dürfen nicht personenbezogen sein oder Rückschlüsse auf Angaben von einzelnen Personen gestatten.

- Der Zugang muß so erfolgen, daß eine Abspeicherung der Daten auf externe Datenträger nicht möglich ist.

Es kann wohl nicht erwartet werden, daß diese Regelung für eine Nutzung des bei der Bundesanstalt „Statistik Österreich“ verfügbaren Datenmaterials durch die Wissenschaft 
förderlich sein wird. Bei personenbezogen Daten wird der Wissenschaft keine Sonderstellung eingeräumt. Wie die Analyse bei sonstigen Daten funktionieren soll, wenn die Daten nicht auf externe Datenträger abgespeichert werden dürfen, ist nicht leicht vorstellbar. Wenn damit gemeint ist, daß die Bundesanstalt „Statistik Österreich“ lokal benützbare Rechenkapazitäten für statistische Analysen von externen Personen bereit stellt, ist zu hoffen, daß auch die wichtigsten Softwarepakete zur Verfügung stehen werden.

Das aus dieser Regelung herauslesbare Mißtrauen gegenüber der Wissenschaft oder die Bevorzugung der Interessen eines rigorosen Datenschutzes gegenüber denen der Wissenschaft kann möglicherweise durch Erfahrungen begründet werden. Daß eine so restriktive Regelung gewählt wurde, ist allerdings aus der Sicht der Wissenschaft enttäuschend und aus volkswirtschaftlicher Sicht unverständlich, wird doch mit dieser Regelung eine effizientere Nutzung des in die Datenerhebung investierten Geldes verhindert. Das Nutzen von personenbezogenen Daten, etwa Überlebensdaten in epidemiologischen Analysen zur Qualitätssicherung in der Medizin wird unmöglich gemacht. Eine Regelung, die durch genaues Protokollieren der Datenzugriffe den Mißbrauch verfolgbar macht, könnte das angestrebte Ziel genauso erreichbar machen.

\section{Abschließende Bemerkungen}

Das BSG 2000 bedeutet für die Amtliche Statistik in vieler Hinsicht eine radikale Änderung und bietet viele Chancen, die zu nützen sind. Ob sie genützt werden können, ist heute schwer abzuschätzen. Ob der fachliche Leiter seiner Verantwortung für ein hohes Qualitätsniveau der statistischen Ergebnisse gerecht werden wird, hängt davon ab, wie sich das Zusammenwirken zwischen ihm und dem kaufmännischen Geschäftsführer und zwischen der Leitung der Bundesanstalt „Statistik Österreich“ und öffentlichen Verwaltung bzw. den zuständigen Politikern entwickeln wird. Die Zukunft wird weisen, ob es der Bundesanstalt „Statistik Österreich“ gelingen wird, zum Träger einer modernen Amtlichen Statistik zu werden.

Adresse der Autoren:

O.Univ.-Prof. Dipl.-Ing. Dr. Peter Bauer Institut für Medizinische Statistik

Universität Wien

Schwarzspanierstraße 17

A-1090 Wien, Österreich

Tel.: +43 1427763200

Fax: +43142779632

Email: Peter.Bauer@univie.ac.at
Univ.-Prof. Dr.h.c.Dr. Peter Hackl

Institut für Statistik

Wirtschaftsuniversität Wien

Augasse 2-6

A-1090 Wien, Österreich

Tel.: +431313364751

Fax: +43131336711

Email: Peter.Hackl@wu-wien.ac.at 